\title{
Evaluation of heat shock protein (HSP-72) expression in retinal ganglion cells of rats with glaucoma
}

\author{
GUO-HUA WANG and YI-QIAO XING \\ Department of Ophthalmology, Renmin Hospital of Wuhan University, Wuhan, Hubei 100853, P.R. China
}

Received January 19, 2017; Accepted April 13, 2017

DOI: $10.3892 /$ etm.2017.4635

\begin{abstract}
The present study was planned to observe the expression of heat shock protein 72 (HSP72) in retina of rats in a glaucoma model. A total of 50 Wistar rats were randomly divided into the high intraocular tension group (glaucoma model) and the sham control (sham operation) group. Glaucoma rat models were created by application of electrocoagulation on at least three groups of veins, reduction of venous return of aqueous humor and by enhancement of intraocular tension at the same time; 1, 2, 3, 4 and 8 weeks after operation, the intraocular tension of rats was observed respectively, and the expression/distribution of HSP72 in retina was assessed by immunohistochemical detection. As a result, the high intraocular tension group was found with obviously increased intraocular tension of the right eyes after operation $(\mathrm{P}<0.05)$, which was stable after one week. It was observed that the positive expression of HSP72 in retina gradually increased significantly with increase in intraocular tension in the rat model of glaucoma in comparison to controls. Furthermore, retinal ganglion cells (RGCs) of rats from both groups were cultured respectively, for confirmation. It was observed that the expression levels of HSP72 in the high intraocular tension group were higher in comparison to the sham control group. In conclusion, the enhanced expression of endogenous HSP72 may play an important role in glaucomatous optic neuro-protection.
\end{abstract}

\section{Introduction}

Glaucoma is one of the prominent eye diseases characterized by continuous increased intraocular tension. Persistent high intraocular tension could damage the eyeball and visual function partially. The patients could even lose complete vision, if left untreated (1). Glaucoma leads to blindness, with total incidence rate of $1 \%$, which gradually increases with age (2). As a severe

Correspondence to: Dr Yi-Qiao Xing, Department of Ophthalmology, Renmin Hospital of Wuhan University, 99 Zhangzhi Dong Road, Wuhan, Hubei 100853, P.R. China

E-mail: gi4024024@163.com

Key words: heat shock protein 72, glaucoma model, ganglion cells eye disease, the increased intraocular tension causes optic disk (named previously as optic nerve head) depression and visual field defect, leading to blindness. The normal intraocular tension ranges from 10-21 mmHg (Schiotz tononometer) and above $24 \mathrm{mmHg}$ is the clear indication of pathological phenomenon. Increased intraocular tension could cause visual function damage with visible typical glaucomatous changes in view (3). The longer the time of increased intraocular tension lasts, the more serious is the visual function damage. The prime reason is the disruption of dynamic equilibrium of the aqueous fluid circulation (4). Heat shock proteins (HSPs) are a kind of heat stress protein among germs and mammals (5). When an organism is exposed to high temperature, the proteins would be compounded and activated for protection. A recent research found that the expression level of HSPs in retina may increase or decrease during the course of glaucoma (6), however, the study of heat shock protein 72 (HSP72) is not clear. The present study analyzed and measured the HSP72 expression of retinal ganglion cells (RGCs) from normal and glaucoma models in rats. It also further verified the specific expression of HSP72 in glaucoma model in rats in terms of protein levels and explored the potential relation between HSP and glaucomatous optic neuropathy.

\section{Materials and methods}

Rats and groups. A total of 50 Wistar rats (Animal Center of Wuhan University, Wuhan, China) of either sex that weighed between 200-300 g were selected and randomly divided into the high intraocular tension group (25 rats) and the sham control group (25 rats). The right eyes were regarded as experimental eyes, while the left eyes as blank control eyes.

Main experimental apparatus. Zeiss ophthalmic operating microscope (Carl Zeiss AG, Oberkochen, Germany), intraocular microscissors, microscopical toothed forceps, underwater electrocoagulator were procured from Karl-Storz GmbH \& Co. KG (Tuttlingen, Germany). Anterior chamber stab knife was from AlconLaboratories, Inc. (Fort Worth, TX,USA) and TONO-PEN XL Tonometer was procured from Mentor Co. (New York, NY, USA). Leica microtome (Leica, Mannheim, Germany) was used with a special in-house no. 9 infusion needle.

Main reagents. HSP72 monoclonal antibody, ready-to-use immunohistochemical Elivision ${ }^{\mathrm{TM}}$ Plus kits of the second 
generation and diaminobenzidine (DAB) chromogenic kit, were procured from Fuzhou Maixin Biological Technology Co., Ltd. (Fuzhou, China).

Establishment of glaucoma models. Related surgical instruments were treated for conventional disinfection. The rats were kept in a quiet and dark environment for a week. Surgical methods: a total of $30 \mathrm{~g} \cdot \mathrm{l}^{-1}$ pentobarbital sodium was used for intra-peritoneal injection anesthesia by $30 \mathrm{mg} \cdot \mathrm{kg}^{-1}$. After anesthesia took effect, the rats were fastened to operating table. A total of $5 \mathrm{~g} \cdot \mathrm{l}^{-1}$ dicaine was dropped to the operative eyes and bulbar conjunctiva was cut $720^{\circ}$ along corneal limbus in order to separate and expose the veins of scleral surface. In the high intraocular tension group, at least three groups of veins from rat sclera surface and corneal limbus peripheral vessels were conducted with electrocoagulation by underwater electrocoagulator, until vascular tissue became white. In the sham control group, cut bulbar conjunctiva without the electrocoagulation of sclera surface veins and corneal limbus peripheral vessels. The operative eyes were smeared with erythromycin eye ointment. After operation, the rats were placed at room temperature and were allowed to wake up naturally. Five days after operation, $2.5 \mathrm{~g} \cdot \mathrm{l}^{-1}$ chloramphenicol eye drops ( 3 times/day) and erythromycin eye ointment (once a day) were used daily. This study was approved by the Animal Ethics Committee of Animal Center of Wuhan University.

Measurement of intraocular tension. The intraocular tension of all rats was measured after 1,2, 3, 4 and 8 weeks of operation, respectively. Inspection methods: a total of $30 \mathrm{~g} \cdot \mathrm{l}^{-1}$ pentobarbital sodium was used for intraperitoneal injection for anesthesia. After anesthesia took effect, the nib of tonometer TONO-PEN XL impinged on cornea and the readings were recorded 3-5 times. The unreliable readings of contact or departure from cornea were discarded and the average was taken as the measured value.

Eyecup preparation. After anesthesia, chest skin and thoracic tissue were cut to expose the heart. Left ventricular was cut off and a no. 9 infusion needle was inserted to aorta ascendens for perfusion. Aorta was clipped by haemostatic forceps and fixed with an infusion needle together. At first, $100 \mathrm{ml}$ normal saline (NS) of $9 \mathrm{~g} \cdot \mathrm{l}^{-1}$ was injected at the speed of $40 \mathrm{ml} \cdot \mathrm{min}^{-1}$, and then about $200 \mathrm{ml}$ (avoiding RGC degeneration) triformol of $40 \mathrm{~g} \cdot \mathrm{l}^{-1}$ was injected quickly until the rats became stiff. Complete eyeballs of rats were taken out and placed into the penicillin bottle with numbers that contained triformol at a dose rate of $40 \mathrm{~g} / \mathrm{l}$. After $30 \mathrm{~min}$, anterior chamber was punctured by stab knife. After $2 \mathrm{~h}$, cornea and crystalline lens were picked out (rats with few vitreous body) to make eyecups. Obvious amotio retinae were avoided when taking out the crystalline lens. Eyecups were put into the same bottle and fixed for 4-6 h. After eyecups were picked out, graded alcohol dehydration, xylene transparency and paraffin embedding were conducted successively for $\mathrm{H} \& \mathrm{E}$ staining and immunohistochemistry.

$H \& E$ staining. Paraffin sections were cut vertically along optic nerve long axis with section thickness of $5 \mu \mathrm{m}$. One section was cut from each paraffin block for H\&E conventional staining.
The organization structure of retina in rats was observed under an optical microscope.

Immunohistochemical staining. Paraffin section proceeded with deparaffination and hydration, which was incubated at room temperature for 10 min after adding $30 \mathrm{gl}^{-1} \mathrm{H}_{2} \mathrm{O}_{2}$, to interdict the activity of endogenous peroxidase. The ready-to-use HSP72 monoclonal primary antibodies were followed by addition of PBS and incubation under $37^{\circ} \mathrm{C}$ for $60 \mathrm{~min}$. Polymer enhancer was added and incubated at room temperature for $20 \mathrm{~min}$. Enzyme-labeled anti-rat/rabbit polymer was added and incubated at room temperature for $30 \mathrm{~min}$. Freshly prepared DAB developing solution was added and color development was performed for 3-10 min, Then followed the treatment with hematoxylin for $40 \mathrm{sec} ; \mathrm{g} \cdot \mathrm{l}^{-1}$ hydrochloric acid alcohol was used for differentiation for $2 \mathrm{sec}$, then washing in water for $15 \mathrm{~min}$. After washing, gradient alcohol dehydration, xylene transparency and neutral balsam mounting were performed step by step.

Counting methods of HSP72 positive cells in retina. The main signal of HSP72 positive expression is brown cytoplasm. Three sections were taken from each paraffin block under 40X objective lens and 5-8 views of retina cells were evenly distributed and selected from each section. Two examiners in the pathological teaching and research office were responsible for the counting of positive and negative cells and for the calculation of positive cell rates.

Western blot analysis. With removed eyeballs and peeled retina, the protein of retina was extracted from the homogenate, and western blot analysis was performed according to the standard protocol. The densities of light bands were analyzed quantitatively by Scion image software (Scion Corp., Frederick, MD, USA).

Statistical analysis. Intraocular tension results were analyzed by SPSS 10.0 (SPSS, Inc., Chicago, IL, USA) one-way ANOVA, and the positive cell rate of retina was detected by $\chi^{2}$. $\mathrm{P}<0.05$ is considered to be statistically significant.

\section{Results}

Detection of intraocular tension after model creation. One week after operation, the intraocular tension was stable, $1,2,3,4$ and 8 weeks after operation, the comparison of intraocular tension among the right eyes of the model group revealed no significant difference $(\mathrm{P}>0.05)$; postoperative right-eye intraocular tensions of the sham control group at different time points were compared with those of its left eyes, without significant difference $(P>0.05)$. At least three groups of veins of rat sclera surface and corneal limbus peripheral vessels were applied for electrocoagulation. The venous return of aqueous humor was reduced and intraocular tension was increased persistently. In this way, the glaucoma model was created successfully (Table I).

Positive expression of HSP72 in retina as detected by immunohistochemical staining. As intraocular tension increased and high intraocular tension persisted, the positive expression 
Table I. Intraocular tensions of different groups of rats $(\mathrm{mmHg})$.

\begin{tabular}{|c|c|c|c|c|}
\hline \multirow{2}{*}{$\begin{array}{l}\text { Operation time } \\
\text { (week) }\end{array}$} & \multicolumn{2}{|c|}{ Increased intraocular tension $(n=25)$} & \multicolumn{2}{|c|}{ Sham operation group $(n=25)$} \\
\hline & Right eyes & Left eyes & Right eyes & Left eyes \\
\hline Baseline & $15.89 \pm 3.16$ & $15.83 \pm 2.83$ & $16.38 \pm 2.87$ & $15.88 \pm 2.63$ \\
\hline 1 & $30.12 \pm 5.18^{a}$ & $15.93 \pm 3.33$ & $16.33 \pm 2.88$ & $15.87 \pm 2.97$ \\
\hline 2 & $32.51 \pm 5.56^{\mathrm{a}}$ & $16.12 \pm 3.11$ & $16.42 \pm 3.28$ & $15.86 \pm 3.14$ \\
\hline 3 & $31.77 \pm 4.43^{\mathrm{a}}$ & $16.32 \pm 3.48$ & $16.33 \pm 3.96$ & $15.94 \pm 2.99$ \\
\hline 4 & $33.07 \pm 5.89^{a}$ & $15.78 \pm 2.48$ & $17.12 \pm 2.92$ & $16.15 \pm 3.23$ \\
\hline 8 & $34.22 \pm 7.28^{a}$ & $16.13 \pm 1.87$ & $17.11 \pm 3.37$ & $16.77 \pm 3.28$ \\
\hline
\end{tabular}

${ }^{\mathrm{a}} \mathrm{P}<0.001$, compared with left eyes of high intraocular tension and binoculus of sham operation group, $1 \mathrm{kPa}=7.5 \mathrm{mmHg}$.

Table II. Positive expression of HSP72 in retina detected by immunohistochemical staining.

\begin{tabular}{|c|c|c|c|c|c|c|c|c|}
\hline \multirow[b]{2}{*}{$\begin{array}{l}\text { Operation time } \\
\text { (week) }\end{array}$} & \multicolumn{2}{|c|}{ Right eyes ${ }^{a}$} & \multicolumn{2}{|c|}{ Left eyes ${ }^{\mathrm{a}}$} & \multicolumn{2}{|c|}{ Right eyes ${ }^{b}$} & \multicolumn{2}{|c|}{ Left eyes ${ }^{b}$} \\
\hline & $\begin{array}{l}\text { Positive } \\
\text { cells }\end{array}$ & $\begin{array}{c}\text { Positive } \\
\text { cell rate }(\%)\end{array}$ & $\begin{array}{l}\text { Positive } \\
\text { cells }\end{array}$ & $\begin{array}{c}\text { Positive } \\
\text { cell rate }(\%)\end{array}$ & $\begin{array}{l}\text { Positive } \\
\text { cells }\end{array}$ & $\begin{array}{c}\text { Positive } \\
\text { cell rate }(\%)\end{array}$ & $\begin{array}{l}\text { Positive } \\
\text { cells }\end{array}$ & $\begin{array}{c}\text { Positive } \\
\text { cell rate }(\%)\end{array}$ \\
\hline 1 & 252 & $16.45^{\mathrm{c}}$ & 26 & 1.76 & 17 & 0.97 & 14 & 0.88 \\
\hline 2 & 367 & $17.91^{\mathrm{c}}$ & 34 & 2.15 & 19 & 0.99 & 16 & 0.93 \\
\hline 3 & 468 & $29.83^{c}$ & 45 & 2.58 & 16 & 1.09 & 17 & 0.96 \\
\hline 4 & 434 & $31.22^{\mathrm{c}}$ & 49 & 2.65 & 21 & 1.35 & 20 & 1.12 \\
\hline 8 & 523 & $41.16^{c}$ & 53 & 3.12 & 22 & 1.6 & 21 & 1.73 \\
\hline
\end{tabular}

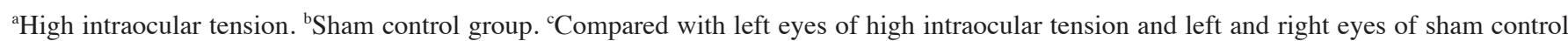
group, $\mathrm{P}<0.001$.

of HSP72 in the retina of the right eyes in the high intraocular tension group mainly manifested in RGCs and retinal nerve fiber layer (RNFL). RGC cytoplasm showed as clay bank and a few RGC colored karyon. There was no obvious positive expression of HSP72 in other layers of retina (Table II, Fig. 1).

Western blot analysis. The expression of HSP72 in high intraocular tension increased by $186 \%$ for the second week, $196 \%$ for the third week and $208 \%$ for the eighth week in comparison with the sham group (Fig. 2). Furthermore, the expression of HSP70 in high intraocular tension increased by $95 \%$ for the second week, $91 \%$ for the third week and $109 \%$ for the eighth week as compared to the sham control group. Protein levels of HSP70 in retina increased by $98.3 \%$ on average, showing that the protein level of HSP70 was unchanged (Fig. 2). Compared with the sham control group, the expression of HSP90 in high intraocular tension increased by $82 \%$ for the second week, $87 \%$ for the third week and $87 \%$ for the eighth week. Protein levels of HSP90 in retina increased by $82.6 \%$ on average, showing that the protein level of HSP90 was unchanged (Fig. 2).

\section{Discussion}

Glaucoma is a serious optic neuropathy. Among the patients with glaucoma, reduction of the intraocular tension could improve the clinical symptom, but optic neuropathy may continue to develop. Research has found that the death mechanism of RGC is RGC apoptosis. Moreover, lack of multiple trophic factors, ischemia and the toxic effect of excitatory amino acid may be the pathology and pathophysiology factors that induce apoptosis (2). Many researchers are seeking for a method that could block or delay the primary and/(or) secondary damage of ganglion cells. At present, the therapeutic methods of glaucoma optic nerve protection include glutamic acid antagonist, calcium antagonist, antioxidant, NO enzyme inhibitor, neuro-trophic factors, vaccination and ganglion cell apoptosis inhibition (7).

HSP is a group of polypeptides with a highly conservative structure. When an organism encounters various stress stimulation, such as ischemia, high temperature, tissue injury and excitatory toxin, leading to the change of gene expression, it induces the generation of HSP, so that the endogenous protection mechanism could be triggered (4). Therefore, HSP is identified as a neuro-protective agent. However, the exact neuro-protective mechanism induced by stress is not clear. According to molecular weight, HSP could be divided into HSP70, HSP90, and micromolecule HSP (8-11). The molecular weight of inducible HSP72 is $72 \mathrm{kDa}$, so it is also known as HSP72, with low expression level in non-stress state, but the expression level would markedly increase in stress state to protect during injury or repair $(4,12,13)$. It has been shown (14) that HSP72 could protect neural pathways 

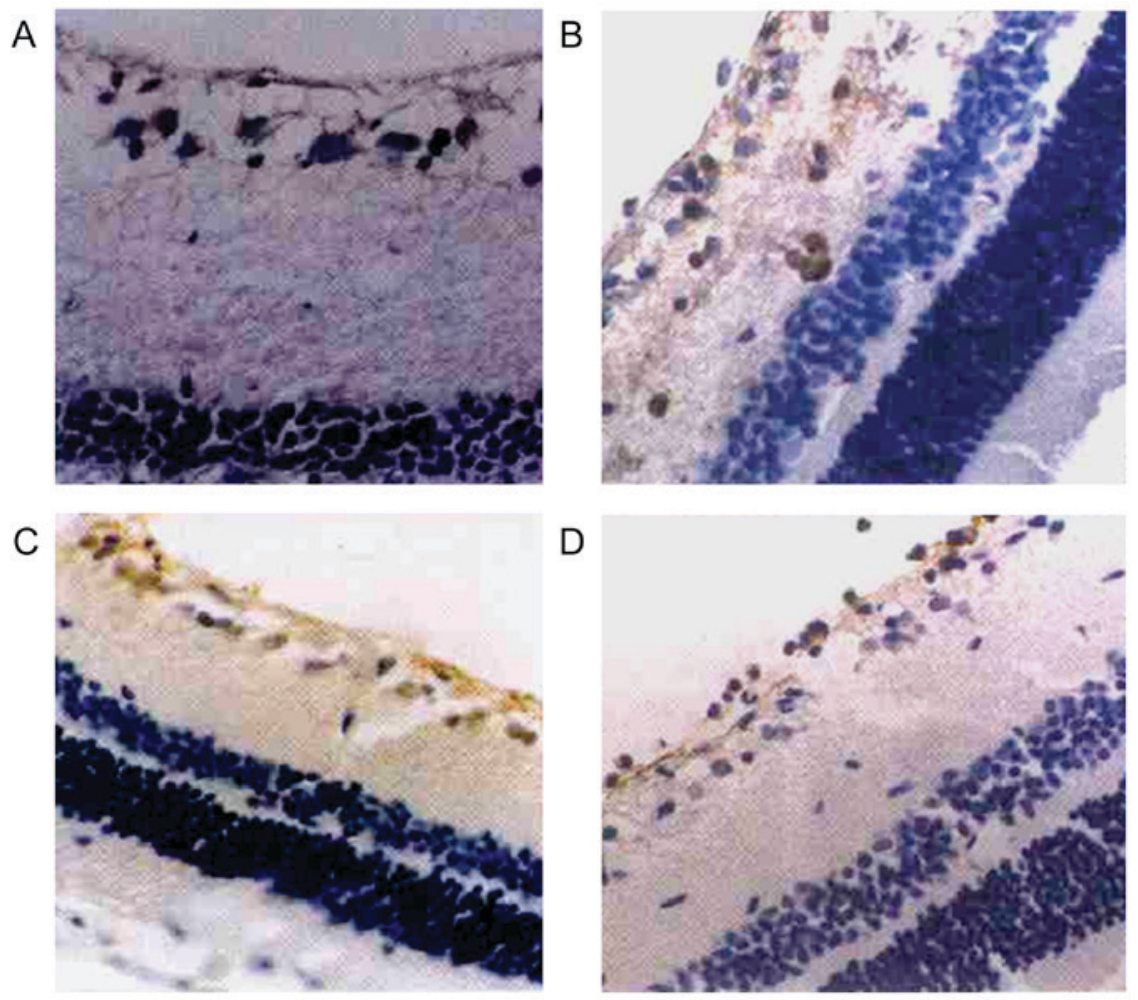

Figure 1. Expression of HSP72 in retina detected by immunohistochemical staining. (A) RGC and RNFL (H\&E magnification, x400) of normal rat retina section under a light microscope. (B) Two weeks after intraocular tension increased, positive expression of HSP72 appeared in partial RGC cytoplasm. (C) Three weeks after intraocular tension increased, RGC with positive expression of HSP72 increased and positive expression of HSP72 also appeared in partial RNFL. (D) Four weeks after intraocular tension increased, RGC with positive expression of HSP72 further increased and partial RNFL showed positive expression of HSP72. HSP72, heat shock protein 72; RGC, retinal ganglion cells; RNFL, retinal nerve fiber layer.

A

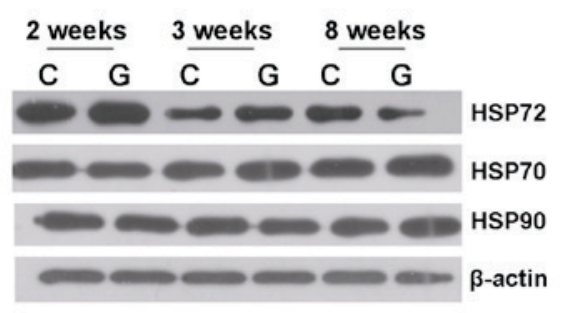

C

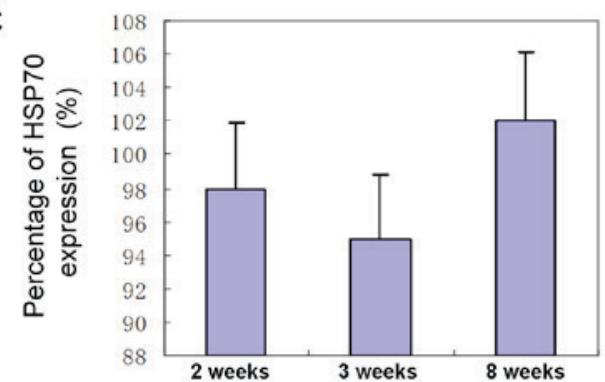

B
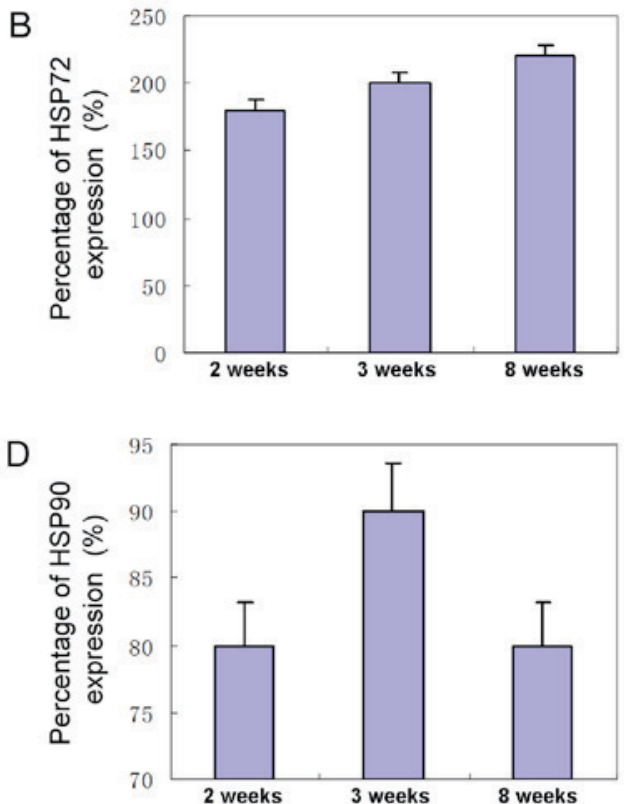

Figure 2. Expression of HSP72, HSP70 and HSP90 in retina detected by immunohistochemical staining. (A) Western blot experiment analysis (C: Sham group; G: High intraocular tension group). (B) The percentage of HSP72 expression level in high intraocular tension retina occupying sham control group in different time points (relative to $\beta$-actin). (C) The percentage of HSP70 expression level in high intraocular tension retina occupying sham control group in different time points (relative to $\beta$-actin). (D) The percentage of HSP90 expression level in high intraocular tension retina occupying sham control group at different time points (relative to $\beta$-actin). HSP72, heat shock protein 72 .

by interdicting the activity of apoptosis pathway SAPK/JNK in RGCs and lateral geniculate body nerve cells (5). HSP72 is considered as an apoptosis inhibitor. Pathological change, namely increased intraocular tension is regarded as one of the 
main risk factors of glaucoma. There are three methods to increase the intraocular tension of glaucoma models experimentally (6). However, most of them could cause obvious inflammatory reaction, with sudden increment for short persistent period. This study applied at least three groups of vortex veins of scleral surface and corneal limbus peripheral vessels by underwater electrocoagulator, so as to observe the influence of intraocular tension on HSP72 as much as possible. After operation, the inflammatory response of operative eyes was light. In addition, the duration of high intraocular tension was long and stable. This basically coincided with the results of increased intraocular tension that applied scleral surface and corneal limbus peripheral vessels by laser photocoagulation $(15,16)$. As intraocular tension increased, high intraocular tension persisted. The high intraocular tension group was found with increased HSP72 expression in RGC of the right eyes and positive cells of RGC (1), but there was no significant difference of HSP72 expression in glaucoma with normal intraocular tension and primary open angle glaucoma. So, the increased expression of HSP72 in glaucoma retina had no relation with intraocular tension. However, it is associated with damage of optic nerve tissue during glaucoma. So, it could be suggested that HSP72 may play a protective role in the injury of glaucoma optic nerve for cells (1). Moreover, the intensity of HSP72 immuno-staining had regional difference in retina, as well as in single RGC. It seems that the priority reaction to stress is in accordance with the mode of optic nerve defect of glaucoma. In the study conducted by Qing et al, the solution of exogenous HSP72 was injected to vitreous cavity of the Wistar rats of ischemia for $60 \mathrm{~min}$ and HSP72 was imported to RGC by electroporation (17). It was observed that exogenous HSP72 could enhance the ability to resist RGC apoptosis induced by ischemia and reperfusion injury. The processing of rat cells with zinc induced generation and secretion of HSP72. This played an important role in rat acute glaucoma model RGCs. So, it could serve as one of the clinical treatment methods for acute glaucoma, and its effective mechanism lies in increasing the expression level of endogenous HSP72.

By creating a glaucoma animal model, this study verified that the expression of HSP72 mainly increased in RGC. Furthermore, the nerve fiber layer of glaucoma may play a protective role for RGC in glaucoma, which will undoubtedly become a significant adjuvant therapeutic measure for glaucoma. Further study of the neuroprotective effect of HSP72 in glaucomatous optic neuropathy may bring about a broad prospect for the effective treatment of glaucoma. Collectively, we believed that the enhanced expression of endogenous HSP72 may play an important role in glaucomatous optic neuro-protection.

\section{References}

1. Sleath B, Sayner R, Vitko M, Carpenter DM, Blalock SJ, Muir KW, Giangiacomo AL, Hartnett ME and Robin AL: Glaucoma patient-provider communication about vision quality-of-life. Patient Educ Couns: Nov 22, 2016 (Epub ahead of print).

2. Shim MS, Kim KY and Ju WK: Role of cyclic AMP in the eye of glaucoma. BMB Rep 50: 60-70, 2017.

3. Giannaccare G, Vagge A, Gizzi C, Bagnis A, Sebastiani S, Del Noce C, Fresina M, Traverso CE and Campos EC: High-intensity focused ultrasound treatment in patients with refractory glaucoma. Graefes Arch Clin Exp Ophthalmol 255: 599-605, 2016.

4. Tosaka K, Mashima Y, Funayama T, Ohtake Y and Kimura I; Glaucoma Gene Research Group: Association between open-angle glaucoma and gene polymorphism for heat-shock protein 70-1. Jpn J Ophthalmol 51: 417-423, 2007.

5. Nowak A, Szaflik JP, Gacek M, Przybylowska-Sygut K, Kamińska A, Szaflik J and Majsterek I: BDNF and HSP gene polymorphisms and their influence on the progression of primary open-angle glaucoma in a Polish population. Arch Med Sci 10: 1206-1213, 2014.

6. Urbak L and Vorum H: Heat shock proteins in the human eye. Int J Proteomics 2010: 479571, 2010.

7. Yokoyama A, Oshitari T, Negishi H, Dezawa M, Mizota A and Adachi-Usami E: Protection of retinal ganglion cells from ischemia-reperfusion injury by electrically applied Hsp27. Invest Ophthalmol Vis Sci 42: 3283-3286, 2001.

8. Birnbaum G: Stress proteins: Their role in the normal central nervous system and in disease states, especially multiple sclerosis. Springer Semin Immunopathol 17: 107-118, 1995.

9. Mehlen P, Schulze-Osthoff K and Arrigo AP: Small stress proteins as novel regulators of apoptosis. Heat shock protein 27 blocks Fas/APO-1- and staurosporine-induced cell death. J Biol Chem 271: 16510-16514, 1996.

10. Yenari MA, Giffard RG, Sapolsky RM and Steinberg GK: The neuroprotective potential of heat shock protein 70 (HSP70). Mol Med Today 5: 525-531, 1999.

11. WoldeMussie E, Ruiz G, Wijono $M$ and Wheeler LA: Neuroprotection of retinal ganglion cells by brimonidine in rats with laser-induced chronic ocular hypertension. Invest Ophthalmol Vis Sci 42: 2849-2855, 2001.

12. Yamashima T: Hsp70.1 and related lysosomal factors for necrotic neuronal death. J Neurochem 120: 477-494, 2012.

13. Sohn S, Im JE, Kim TE and Kee C: Effect of heat shock protein 72 expression on etoposide-induced cell death of rat retinal ganglion cells. Korean J Ophthalmol 27: 48-51, 2013.

14. Li N, Li Y and Duan X: Heat shock protein 72 confers protection in retinal ganglion cells and lateral geniculate nucleus neurons via blockade of the SAPK/JNK pathway in a chronic ocular-hypertensive rat model. Neural Regen Res 9: 1395-1401, 2014.

15. Tezel G, Hernandez R and Wax MB: Immunostaining of heat shock proteins in the retina and optic nerve head of normal and glaucomatous eyes. Arch Ophthalmol 118: 511-518, 2000.

16. Nickells RW: Retinal ganglion cell death in glaucoma: The how, the why, and the maybe. J Glaucoma 5: 345-356, 1996.

17. Qing G, Duan X and Jiang Y: Induction of heat shock protein 72 in RGCs of rat acute glaucoma model after heat stress or zinc administration. Yan Ke Xue Bao 20: 30-3, 2004. 